\title{
External Magnetic Field Measurements in the UME Kibble Balance
}

\author{
Hacı Ahmedov ${ }^{1}$, Beste Korutlu ${ }^{1}$, Lev Dorosinskiy ${ }^{1}$, Recep Orhan ${ }^{1}$, Özlen Tuncel ${ }^{1}$ \\ ${ }^{1}$ TÜBITAK National Metrology Institute (UME), Kocaeli, TURKEY
}

\begin{abstract}
The new definition of the kilogram in terms of the fixed value of Planck's constant ensures the long-term stability of the SI mass unit and enables traceability from more than one source. Kibble balance experiments offer an effective primary realisation method for the new definition of a kilogram. The Kibble balance apparatus at the National Metrology Institute of Turkey is designed with a stationary coil and an oscillating magnet. In contradistinction to traditional moving coil Kibble balance experiments, the external magnetic field introduces an asymmetry between the Ampere's law of force and the Faraday's law of induction in moving magnet experiments. In this paper, we develop a method based on external magnetic flux density difference measurements in a vertical direction by using a magnetic gradiometer to take into account the effect of the external magnetic field on the realisation of kilogram. We have examined the time variations of the external magnetic flux differences and non-liner effects to guarantee the validity of the model. The proposed model fits well with the data, and the kilogram realisation requirement is fulfilled within the accuracy level of the electrical measurements taken. We conclude that, when placed in close proximity to the Kibble balance, the magnetic gradiometer may be used for monitoring purposes.
\end{abstract}

\section{Section: RESEARCH PAPER}

Keywords: Kibble balance; magnetic flux density; measurement techniques; Planck's constant

Citation: Haci Ahmedov, Beste Korutlu, Lev Dorosinskiy, Recep Orhan, Özlen Tuncel, External Magnetic Field Measurements in UME Kibble Balance, Acta IMEKO, vol. 9, no. 3, article 6, September 2020, identifier: IMEKO-ACTA-09 (2020)-03-06

Editor: Jan Saliga, Technical University of Košice, Slovakia

Received January 17, 2020; In final form March 24, 2020; Published September 2020

Copyright: This is an open-access article distributed under the terms of the Creative Commons Attribution 3.0 License, which permits unrestricted use, distribution, and reproduction in any medium, provided the original author and source are credited.

Funding: This work was supported by TÜBITAK National Metrology Institute, Turkey.

Corresponding author: Haci Ahmedov, e-mail: haji.ahmadov@tubitak.gov.tr

\section{INTRODUCTION}

The redefinition of the kilogram unit, approved at the $26^{\text {th }}$ meeting of The General Conference on Weights and Measures (CGPM), held in November 2018, has replaced its previous artefact definition via the mass of the International Prototype of the Kilogram (IPK) with one based on the fixed numerical value of the Planck constant [1], [2]. Extensive studies have been performed at several national metrology institutes (NMIs) across the world to ensure a smooth transition to the revised definition of the kilogram. Currently, there are two independent primary methods for realising the kilogram with an uncertainty of a few parts in $10^{8}$ : Kibble balance [3]-[23] and X-ray-crystal density (XRCD) [24]-[29]. The content of this paper draws upon the Kibble balance principle. Originally devised at the National Physical Laboratory (NPL) by Brian Kibble in 1975 [30], [31], the Kibble balance relates mechanical and electrical powers where the electrical power is measured in terms of Planck's constant by using two macroscopic quantum phenomena known as the quantum Hall effect [32] and the Josephson effect [33] to link the macroscopic mass to the Planck constant. A number of Kibble balances with different geometries and experimental protocols have been constructed in various NMIs [3]-[23]. The National Metrology Institute of Turkey (UME) has constructed a Kibble balance with a stationary coil suspended from the load cell of a mass comparator and a surrounding oscillating magnetic circuit. The prominent features of the UME Kibble balance can be seen in the adopted novel measurement procedure whereby the oscillating parameters are continuously averaged over the magnet oscillation half-cycles (see [34]-[37] for details of the adopted measurement procedure). As a result, the variations in magnetic field and temperature are suppressed, which in turn enables the construction of both the magnetic circuit and the apparatus in smaller dimensions. The oscillatory motion of the magnetic circuit also allows the simultaneous operation of weighing and moving phases (i.e. concurrent testing of Ampere's force law and Faraday's law of induction), which eliminates the need to 
precisely quantify the variations in the environmental and experimental conditions between the two phases. In addition, the system does not require a fine adjustment between the magnetic centre of the magnetic circuit and electric centre of the coil. Instead, the misalignment is treated as a parameter contributing to the Faraday's Voltage across the ends of the coil generated due to the relative motion between the coil and magnetic circuit [36], [37]. Aside from its distinctive properties, there is an important difference between the UME Kibble balance and traditional twophase, moving coil Kibble balances in that the external magnetic field introduces an asymmetry between the Ampere's law of force and Faraday's law of induction. In the present work, we develop a convenient approach for taking into account the effect of Earth's magnetic field on the oscillating magnet Kibble balance. The approach guarantees the desired uncertainties in the realisation experiment within the accuracy level of electrical measurements.

In Section 2, the asymmetry between Faraday's Law of Induction and Ampere's Force Law in relation to the moving magnet is described. In Section 3, the UME Kibble balance external magnetic force measurements are presented, in which the importance of the stability of external magnetic flux density difference and non-linear effects is emphasised. In Section 4, the monitoring of the magnetic flux density is explained. Finally, we offer some conclusions in Section 5.

\section{ASYMMETRY IN MOVING MAGNET KIBBLE BALANCES}

Currently, in all Kibble balance experiments, a permanent magnetic circuit is adopted as the most practical solution when balancing the mechanical power with the electrical one. Originally designed by the International Bureau of Weights and Measures (BIPM) [38], a typical symmetrical permanent magnetic circuit is widely used by worldwide Kibble balance experiments. Generally, SmCo is used as the permanent magnetic material since it has high performance and a low temperature coefficient. Thanks to the magnetic circuit, the magnetic flux density generated by the permanent magnetic material is directed towards the narrow air gap around the coil parallel to the inner and outer soft-iron yokes. Thus, a strong, vertically uniform, radially magnetic field is generated in the vicinity of the coil [39]. The cross-sectional view of the UME permanent magnetic circuit is given in Figure 1.

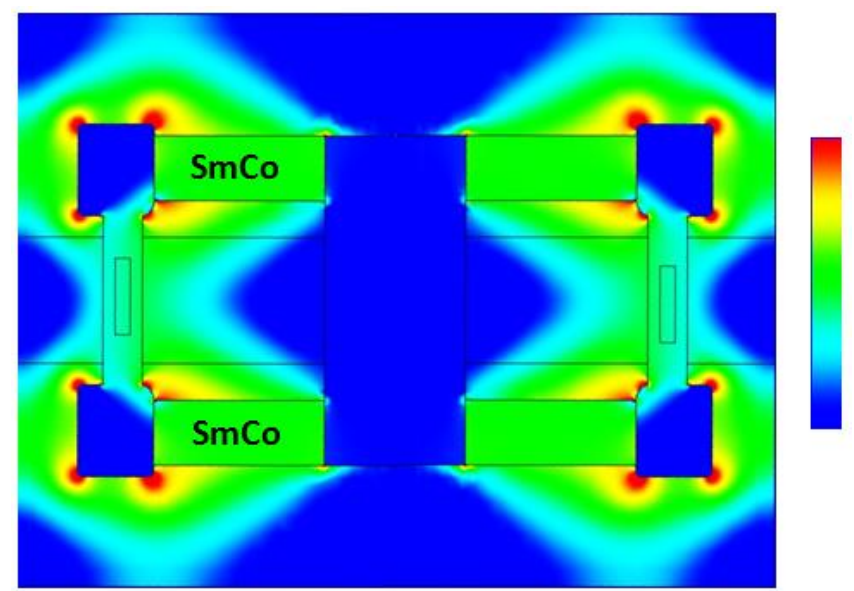

Figure 1. Cross-sectional view of the UME magnetic circuit. The yoke is made from iron, and the permanent magnets are made from SmCo. The colour scale in the colour bar goes from high magnetic flux density given in red to low given in dark blue.
The coil located in the air gap of the magnetic circuit is stationary as it is suspended from the load cell of the mass comparator. The magnetic circuit, on the other hand, oscillates in the direction of the gravitational acceleration. The soft-iron yoke not only guides the generated magnetic flux density towards the coil volume but also effectively shields the coil from the effect of external magnetic flux density. The exposure of the yoke itself, a magnetically non-linear material, to the external magnetic flux density gives rise to an additional magnetic flux density in the air gap, which is called the magnetisation flux density. The magnetisation flux density compensates for the external magnetic flux density in the air gap, which is the manifestation of the magnetic shielding principle for cavities in magnetic materials.

In moving magnet Kibble balance experiments, there occurs an asymmetry between Ampere's law of force and Faraday's law of induction due to the external magnetic field. We describe this asymmetry in the following sections.

\subsection{Ampere's Law of Force}

There are three sources of magnetic flux density created in the air gap:

a) magnetic flux density generated by the magnetic circuit $\left(\vec{B}_{\mathrm{mc}}\right)$,

b) magnetisation flux density $\left(\vec{B}_{\mathrm{m}}\right)$,

c) external magnetic flux density $\left(\vec{B}_{\mathrm{e}}\right)$,

which contribute to the Ampere's force law given by $\vec{F}=$ $J \oint d \vec{l} \times \vec{B}$, where $\vec{B}=\vec{B}_{\mathrm{mc}}+\vec{B}_{\mathrm{m}}+\vec{B}_{\mathrm{e}}$ is the total magnetic flux density in the air gap, $J$ is the DC current on the coil and $\vec{l}$ is the vector having a magnitude equal to the total length of the coil and being in the direction of electric current $J$.

The force on the coil in the direction of gravitational acceleration is obtained by the $\operatorname{dot}$ product $F=\hat{n} \cdot \vec{F}$, where $\hat{n}$ is the unit vector representing the direction of gravitational acceleration yielding.

$$
F=F_{\mathrm{mc}}+F_{\mathrm{m}}+F_{\mathrm{e}} \text {. }
$$

Here, $F_{m c}$ is the force generated by the magnetic flux density of the magnetic circuit, and $F_{\mathrm{m}}$ and $F_{\mathrm{e}}$ are the forces induced on the coil by the magnetisation and external magnetic flux densities, respectively.

\subsection{Faraday's Law of Induction}

As the coil is stationary with respect to the external magnetic flux density, only $\vec{B}_{\mathrm{mc}}$ and $\vec{B}_{\mathrm{m}}$ contribute to the Faraday's law of induction. As a result, for an oscillating-magnet Kibble balance, the fundamental equation takes the following form:

$$
\frac{h}{h_{\mathrm{c}}} V=\frac{F-F_{\mathrm{e}}}{J} u \text {, }
$$

where $V$ is a Faraday's voltage across the ends of the coil and $u$ is the velocity of the coil with respect to the magnet. Here $h$ is the Planck constant and $h_{\mathrm{c}}$ is the consensus value of the Planck constant [2]. The ratio $h / h_{\mathrm{c}}$ appears since the Faraday's voltage is measured in terms of the consensus value of the Planck constant by using the Josephson Effect. In the Kibble balance experiment, the quantities measured directly are the total vertical force $F$, the electrical current $J$, the vertical velocity $u$ and the Faraday voltage $V$. The force produced by external magnetic field $F_{\mathrm{e}}$ on the other hand is not a directly measured quantity in the 
Kibble balance experiment, and an indirect measurement procedure should be developed to take this factor into account. One way to handle this effect is put forward by the Chinese Joule Balance team, in which they place two compensation coils connected in series with the suspended coil in the air gap of the permanent magnet system. One of the compensation coils is fixed to the inner yoke while the other one is fixed to the outer yoke. The number of turns in each compensation coils is equal to half of the number of turns of the suspended coil. When the magnet moves, the compensation coils move together with the magnet. Thus, the relative motion between the external magnetic field and the compensation coils induces Faraday's voltage across the ends of the compensation coils. As they are connected in series with the suspended coil, the asymmetry in (2) is eliminated. This is ensured by placing the compensation coils sufficiently close to the suspended coil. Although the idea of compensation coils is neat, mechanical difficulties may arise while placing three coils in the air gap [40], [41].

In this paper, we propose another method for taking into account the effect of an external magnetic field on a stationary coil and oscillating magnet system without compensation coils. One may rewrite (2) as

$$
\frac{h}{h_{\mathrm{c}}}=Q \frac{\langle F\rangle}{\langle J\rangle}\{u \mid V\},
$$

by following the dynamical measurement procedure of the UME Kibble balance [34], [35], where the integration time is chosen to be a multiple of oscillation fundamental period $T$ such that $\tau=$ $N T$. Here $\langle F\rangle$ and $\langle J\rangle$ are the average of the total Ampere's force on the coil and current over the integration time, respectively, $\{u \mid V\}$ represents the average of the velocity $u$ of the coil with respect to the magnet over the half-cycles of Faraday's voltage $V$, and $Q$ is the geometric factor, which includes inhomogeneities and the effect of external magnetic flux density on the Planck constant. Using (3), the effect of external magnetic field on the Planck constant is found to be

$$
q=-\frac{\left\langle F_{\mathrm{e}}\right\rangle}{\langle F\rangle},
$$

where $\left\langle F_{\mathrm{e}}\right\rangle$ is the average of the force induced on the coil by the external magnetic flux density over the integration time. Practically speaking, the force created by the external magnetic flux density is measured when there is no magnetic circuit, while Kibble balance measurements are performed when the coil is surrounded by the magnetic circuit. The two conditions for this method to be valid are:

a) The variations of $F_{\mathrm{e}}$ in time should be small enough to be negligible.

b) The nonlinear effects caused by the interaction of the magnetic circuit with external bodies should be small enough to be negligible.

If these conditions are met, there will be no need for compensation coils. We clarify this statement in the next section.

\section{EXTERNAL MAGNETIC FORCE MEASUREMENTS}

The schematic circuit diagram of the measurement set-up for external magnetic force measurements is given in Figure 2. Below we give a step-by-step description of the research conducted.

1. The $q$ factor in (4) is determined by simultaneous measurement of $F_{\mathrm{e}}$ and $J$ when there is no magnetic circuit around the coil. $F_{\mathrm{e}}$ is determined by mass measurements with a mass comparator, and the current is determined by measuring the voltage across the ends of a standard resistor connected to the coil in series.

2. The time variations of $F_{\mathrm{e}}$ are determined by measuring external magnetic flux density differences via magnetic gradiometer. Over the short term, the variations in $F_{\mathrm{e}}$ are found to be negligible.

3. The possible non-linear effects when the magnetic circuit is placed around the coil are simulated by EMS Magnetic Software. They are found to be small enough to disregard.

4. The correlation between $F_{\mathrm{e}}$ and the magnetic flux density difference measurements by the gradiometer sensor is determined when an additional axial magnetic field source is used to simulate abrupt changes in external magnetic field. Once the correlation coefficient is determined, the magnetic gradiometer can be used for monitoring the variations in $F_{\mathrm{e}}$.

It is important to emphasise that there is no magnetic circuit surrounding the suspended coil. Therefore, the Ampere's force in (1) reduces to $F=F_{\mathrm{e}}$. The force measurements are carried out with the load cell of an AX5006 mass comparator with $1 \mu \mathrm{g}$ resolution that is integrated into the UME Kibble Balance. DC current is measured by Keysight 3458 A digital multimeter across the two Tinsley 5658A $100 \Omega$ standard resistors connected in parallel and placed in an oil bath. The external magnetic flux density difference measurements are performed with a Bartington Grad601 magnetic gradiometer, which will be explained in the following subsections. DC calibration of the Keysight 3458 A digital multimeter with Programmable Josephson Voltage Standard (PJVS) yields a total relative measurement uncertainty of $0.1 \mathrm{ppm}$. The uncertainty components in the force, the standard resistance and the change in magnetic flux density difference measurements are small compared to the uncertainty from to the digital multimeter. Therefore, the total uncertainty is restricted by the uncertainty in the $\mathrm{DC}$ voltage measurements.

Figure 3 illustrates the ratio of the force by the external magnetic flux density $F_{\mathrm{e}}$ and the DC current $J$ averaged every 10 minutes. There is a $10 \mathrm{~min}$. delay between each consecutive piece of data. The current direction has been reversed every $100 \mathrm{~s}$ to handle the drifts in the balance.

We obtain the mean value of the ratio as $\left\langle F_{\mathrm{e}} / J\right\rangle=$ $-0.295 \mathrm{mN} / \mathrm{A}$ and the standard deviation as $\sigma\left(F_{\mathrm{e}} / J\right)=$ $0.005 \mathrm{mN} / \mathrm{A}$. Since the geometrical factor $\langle F / J\rangle$ in our experiment is about $150 \mathrm{~N} / \mathrm{A}$, using (4), we obtain $q_{\mathrm{m}}=$ $1.97 \times 10^{-6}$ with a standard deviation of $3 \times 10^{-8}$.

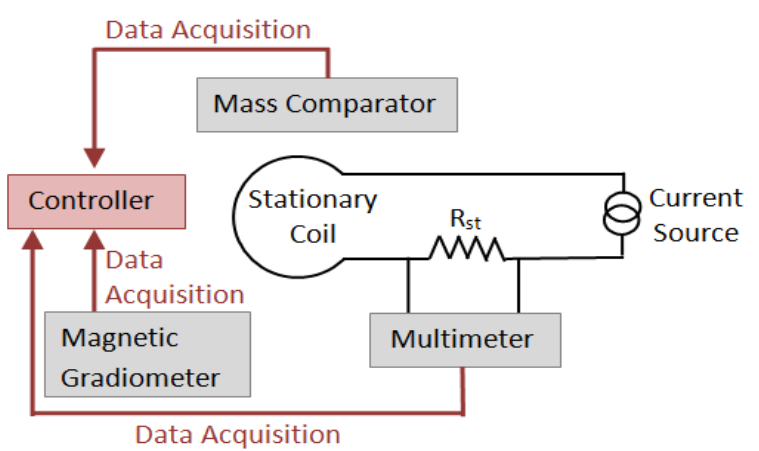

Figure 2. The schematic circuit diagram of the external magnetic flux density measurement procedure. 


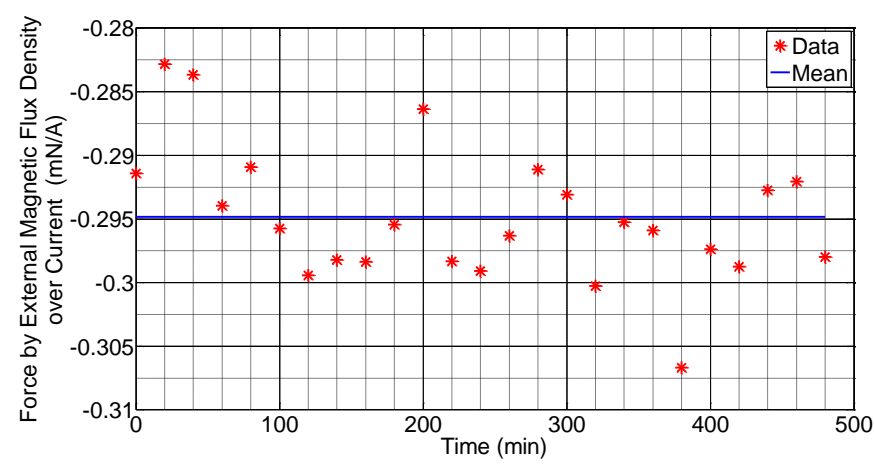

Figure 3. The ratio of the force by the external magnetic flux density and the DC current averaged every $10 \mathrm{~min}$. There is a $10 \mathrm{~min}$. delay between each consecutive piece of data. The current direction has been reversed every 100 $\mathrm{s}$ to account for drifts in the balance. The mean value of the ratio $\left\langle F_{\mathrm{e}} / J\right\rangle=$ $-0.295 \mathrm{mN} / \mathrm{A}$ is given in the blue straight line. The standard deviation is $\sigma\left(F_{\mathrm{e}} / J\right)=0.005 \mathrm{mN} / \mathrm{A}$

\subsection{Stability of External Magnetic Flux Difference}

As emphasised in Section 2, the variation of $F_{e}$ in time should be small enough to allow the proposed procedure to be followed by taking into account the effect of the external magnetic flux density. One may easily find that the vertical component of the Ampere's force is proportional to the magnetic flux through the lateral surface of the coil by using simple vector algebra. Based on the fact that the divergence of magnetic flux density over a closed surface is equal to zero, the Ampere's force is expressed in terms of the magnetic flux difference $\delta \Phi$ between the top and the bottom surfaces of the coil as

$$
F_{\mathrm{e}}(t)=\frac{J M \delta \Phi_{\mathrm{e}}(t)}{d}
$$

where $d$ is the height of the coil and $M$ is the number of turns in the coil. Substituting (5) in (4) yields

$$
q=-\frac{\left\langle\delta \Phi_{\mathrm{e}}\right\rangle}{\langle\delta \Phi\rangle}
$$

where the effect of the external magnetic field on the Planck constant is reflected in the ratio of the flux differences instead of the forces. We estimate $\langle\delta \Phi\rangle$ as the product of the radial magnetic flux density $\langle\delta B\rangle$ in the centre of the air gap and the lateral area of the coil, while $\left\langle\delta \Phi_{\mathrm{e}}\right\rangle$ is estimated by the product of external magnetic flux density difference $\left\langle\delta B_{\mathrm{e}}\right\rangle$ and the

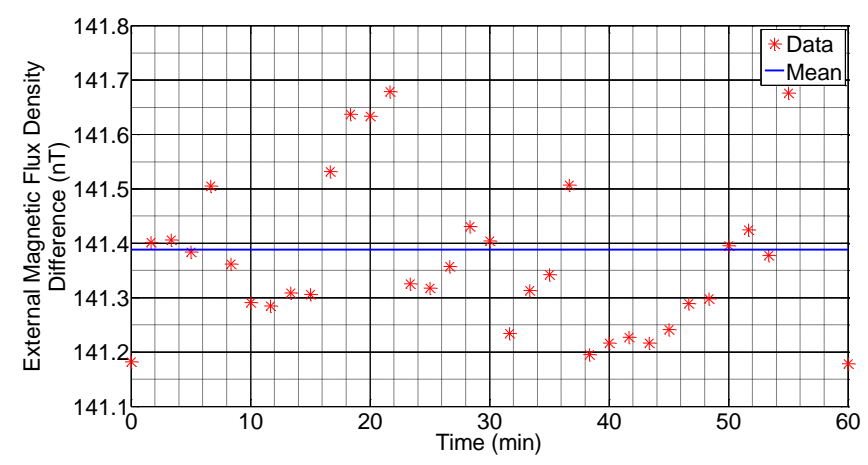

Figure 4. The temporal changes of the difference of external magnetic flux density between the two sensors of the fluxgate gradiometer averaged in every $100 \mathrm{~s}$. The mean value of external magnetic flux density difference $\left\langle\delta B_{\mathrm{e}}\right\rangle=141.388 \mathrm{nT}$ is given in blue. The standard deviation is $\sigma\left\langle\delta B_{\mathrm{e}}\right\rangle=$ $0.160 \mathrm{nT}$.

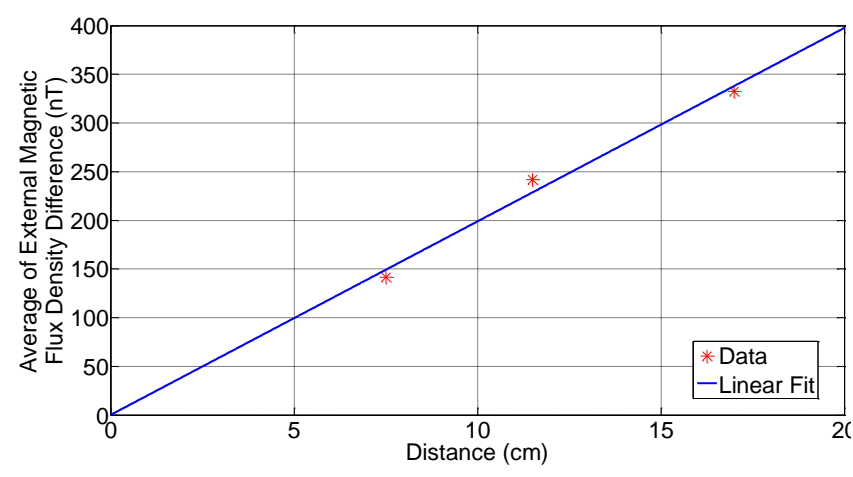

Figure 5. The average of external magnetic flux density difference with respect to the distance between the sensors.

horizontal area of the coil. Thus, one may write $q \propto$ $(r / 2 d)\left\langle\delta B_{\mathrm{e}}\right\rangle /\langle\delta B\rangle$, where $r$ is the radius of the coil. The height of the coil is around $2 \mathrm{~cm}$ and radius is around $7 \mathrm{~cm}$. The radial magnetic flux density in the centre of the air gap is around 0.5 $\mathrm{T}$. Thus, if the variations of $\left\langle\delta B_{\mathrm{e}}\right\rangle$ are smaller than $0.5 \mathrm{nT}$, we may neglect the variations in q-factor. We use a commercial Bartington single-axis vertical component fluxgate gradiometer for flux density difference measurements whereby two cylindrical gradiometer sensors are mounted on a rigid beam fixed at a distance of $90 \mathrm{~cm}$. We modified the rigid beam so that two sensors could be fixed at different positions. The minimum possible distance between the two sensors is $7.5 \mathrm{~cm}$ after this modification. Figure 4 demonstrates the temporal changes in the difference of the external magnetic flux density between the two sensors of the fluxgate gradiometer averaged every $100 \mathrm{~s}$, where the sensors are separated at a distance of $7.5 \mathrm{~cm}$. The mean value of the temporal changes in the difference of external magnetic flux density between the two sensors is $\left\langle\delta B_{\mathrm{e}}\right\rangle=141.388 \mathrm{nT}$, and the standard deviation is $\sigma\left\langle\delta B_{\mathrm{e}}\right\rangle=0.160 \mathrm{nT}$.

The linearity tests performed at three different distances between the sensors of the magnetic gradiometer are given in Figure 5.

Now that the linearity is observed, it is possible to interpolate the standard deviation obtained by the fluxgate gradiometer at the minimum distance between the sensors of the fluxgate gradiometer to the one over the top and bottom surfaces of the coil by linear approximation, which yields $\sigma\left\langle\delta B_{\mathrm{e}}\right\rangle \sim 0.05 \mathrm{nT}$.

\subsection{Non-linear Effects}

Since the effect of the external magnetic flux on the Planck constant is determined in the absence of the magnetic circuit, it is important to make the possible non-linear effects generated in the presence of the magnetic circuit negligible. The non-linear effects include the influence of the magnetic circuit on the nearby soft magnetic materials, which in turn is reflected in the external magnetic flux. This can be achieved by keeping the magnetic materials away from the permanent magnet. The simulations are carried out in the EMS Magnetic Software to support this idea (see Figure 6).

We simulated the non-linear effect of the external magnetic flux by using ferromagnetic material of a cylindrical form with a diameter of $20 \mathrm{~cm}$ and height of $10 \mathrm{~cm}$. The distance between the center of the ferromagnetic material and the magnetic circuit was taken to be $62.5 \mathrm{~cm}$. The centre of the ferromagnetic material is placed on the same symmetry axis as the magnetic circuit. We use pure iron as the ferromagnetic material. The 


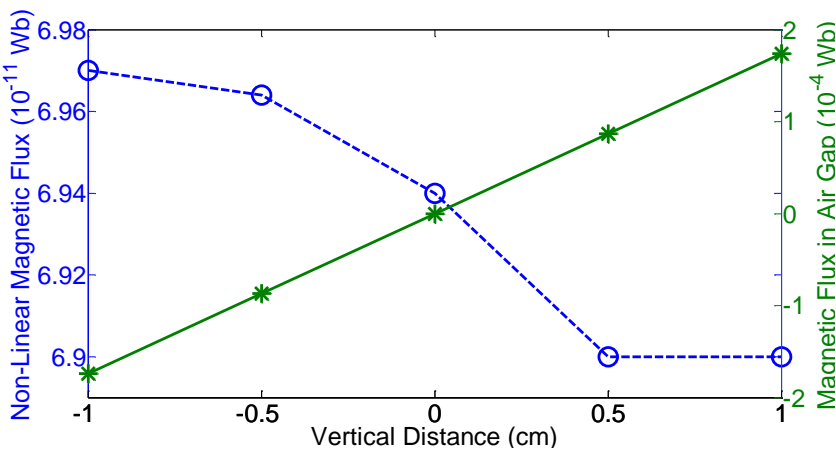

Figure 6. The simulation carried out in the EMS Magnetic Software. Given in blue dashed line with circle markers are the non-linear effects of external magnetic field and in green solid line with star markers indicate the magnetic flux in the air gap.

magnetic circuit oscillates vertically around the centre of the coil with an amplitude of $1 \mathrm{~mm}$. The vertical distance given in the $\mathrm{x}$ axis of Figure 6 represents the distance between the centre of the magnetic circuit and the centre of the coil. As the height of the coil is around $d=2 \mathrm{~cm}$, we simulated the region within a displacement of $\pm 1 \mathrm{~cm}$ from the origin where the two centres coincide. The flux through the horizontal disk with a radius of $7 \mathrm{~cm}$ is simulated. The y-axis on the right represents the flux in the air gap for vertical displacements of $\pm 1 \mathrm{~cm}$ from the origin, while the one on the left represents the differences between the flux in the presence and in the absence of ferromagnetic material. The effect of the non-linear flux is estimated to be $3 \times 10^{-9}$. It is important to note that in the actual experiment we expect the non-linear effects to be much smaller as there would be no heavy ferromagnetic materials near the magnetic circuit.

\section{MONITORING THE CHANGE IN MAGNETIC FLUX DENSITY}

The external magnetic flux density differences are measured on different days. In normal conditions, we observe that the time variations in external magnetic flux density differences are negligible. However, in case of geophysical natural phenomena, the variations may be high. In addition, moving heavy magnetic materials near the Kibble balance system may cause additional variations in the external magnetic field since the magnetic circuit may magnetise these materials, and in turn, they may contribute to the external magnetic field. This is why monitoring of the external magnetic flux density differences may be necessary. The temporal changes in the flux through the suspended coil may be characterised by using the magnetic gradiometer locally. This is possible since the characteristic scales of the factors mentioned above that produce variations in external magnetic flux density are very large compared to the distance between the coil and the magnetic gradiometer sensors. Therefore, we expect the Ampere's force on the suspended coil to be strongly correlated with the magnetic flux density difference measured by the magnetic gradiometer sensors. Using (5), we may write this correlation in the following form

$$
\left\langle F_{\mathrm{e}}\right\rangle=\zeta\langle\rangle\left\langle\delta B_{\mathrm{e}}\right\rangle,
$$

where $\zeta$ is a model parameter that depends on the geometry of suspended and sensor coils and also on the local inhomogeneities in the external magnetic flux density differences. We determine the value of the model parameter $\zeta$ by minimising the quadratic form

$$
\mathfrak{F}(\zeta)^{2}=D\left(\left\langle F_{\mathrm{e}}\right\rangle-\zeta\langle J\rangle\left\langle\delta B_{\mathrm{e}}\right\rangle\right),
$$

where $D$ is the standard deviation. Since the target uncertainty in the realisation of a kilogram is twenty parts per billion, the modelling given in (7) will meet the requirements provided that $\mathfrak{F} /\langle F\rangle$ is much less than $10^{-8}$. It is important to note that the model parameter $\zeta$ is determined in the absence of a magnetic circuit. The presence of a magnetic circuit may directly affect the gradiometer sensors such that an offset may occur. However, it is possible to determine this offset independently by measuring the difference in the presence and absence of the magnetic circuit. Then, $\delta B_{\mathrm{e}}$ will be modified accordingly in the optimisation procedure. In this way, using (7), we will be able to trace the variations of the $q$-factor.

The asymmetry between Faraday's Law and Amperes Law can be treated with compensating coils, as proposed by the NIM Joule Balance Group [40], [41]. We have introduced an alternative approach where, under certain circumstances, the asymmetry could be accounted for without compensation coils by means of a magnetic gradiometer. However, we are planning to use both the compensating coils and the proposed magnetic gradiometer method to increase the reliability of the results. The comparative analysis between these two methods will be conducted after the ongoing modifications on the handler of the mass comparator are complete.

We demonstrate the correlation in (7) with the help of an additional axial magnetic field source, which is introduced to simulate the effect of possible extreme variations in the external magnetic field due to the reasons mentioned above. The source is composed of a pair of identical square coils with a side length of $1.5 \mathrm{~m}$ separated at a distance equal to the side length of the square that are used to create an additional axial magnetic flux density, $B_{\text {axial }}$. In this way, we produce short term variations of the external magnetic flux density of a sufficient magnitude to be measured by magnetic gradiometer. A magnetic flux density of 1 Gauss is produced at the center of the coil pair provided that they are connected in series. The coil is placed next to the UME Kibble balance apparatus. For our purposes, $I=4 \mathrm{~A}$ is switched on and off every $10 \mathrm{~min}$ to create this additional flux density where $B_{\text {axial }}=0$ is equivalent to the case of the coil under the sole influence of the external magnetic flux density. In Figure 7 we show the temporal changes of the difference in magnetic flux density averaged every $10 \mathrm{~min}$ in the presence and absence of the axial flux density. It is clear from Figure 7 that the variations in external magnetic field density difference can be traced by the magnetic gradiometer. The apparent difference of approximately $400 \mathrm{nT}$ in the external magnetic flux density in the presence

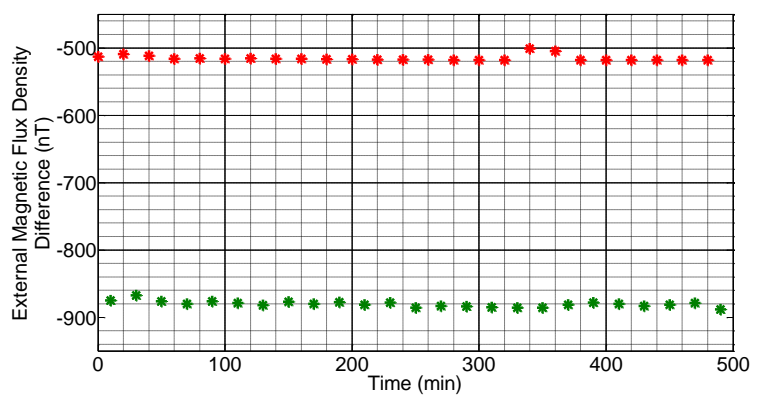

Figure 7. The temporal changes of the external magnetic flux density difference averaged in every 10 min when the axial field is on and off. Given in red star is given the actual data with $B_{\text {axial }}=0$ and in green star is with $B_{\text {axial }} \neq 0$. The separation between the sensors of gradiometer is $90 \mathrm{~cm}$. 


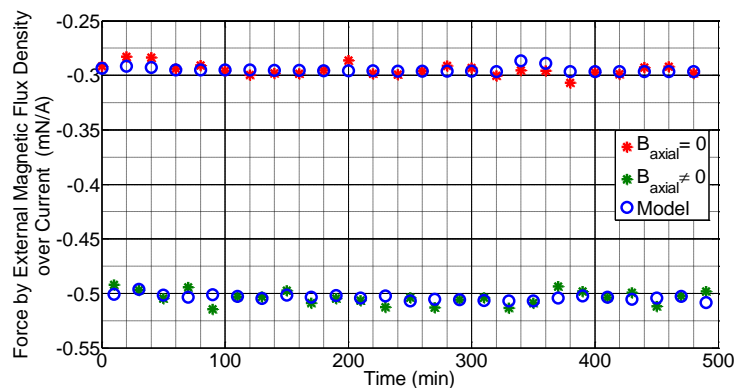

Figure 8. Fitting of the data by minimisation of the quadratic form in (6). Given in red star is given the actual data with $B_{\text {axial }}=0$, in green star is with $B_{\text {axial }} \neq 0$ and in blue circles is the model fitting data with $\zeta=583.7 \mathrm{~m}$. The separation between the sensors of gradiometer is $90 \mathrm{~cm}$.

(lower data) and in the absence of the axial field (upper data) allows the Ampere's force measurements, which otherwise cannot be detected within its resolution, to be monitored with the mass comparator.

With this data at hand, we solve for the optimisation parameter $\zeta$ by minimising the quadratic form in (6), which yields $\zeta=583.7 \mathrm{~m}$. The actual data and model fitted data of the ratio $\left\langle F_{\mathrm{e}}\right\rangle /\langle\mathrm{J}\rangle$ are given in Figure 8.

Additionally, the actual data for $B_{\text {axial }}=0$ and $B_{\text {axial }} \neq 0$ are shown as red and green stars, respectively, and the model fitting data using $\zeta=583.7 \mathrm{~m}$ is shown as blue circles. The actual data and the fitted data coincide with each other. This is an indication of the strong correlation (0.9986) between the force from the external magnetic flux density over the current and the magnetic flux density differences. The target accuracy in (7) is achieved since we obtain $\mathfrak{F} /\langle F\rangle=4 \times 10^{-9}$.

\section{CONCLUSION}

To deal with the impact of the external magnetic field on the determination of the Planck constant within a Kibble balance experiment, we have proposed an approach based on vertical external magnetic flux density difference measurements. The proposed method offers a convenient and practical solution for taking into account the effect of Earth's magnetic field on the moving magnet in Kibble balance experiments. A magnetic gradiometer placed in close proximity to a Kibble balance with a moving magnet may be used for monitoring the variations in external magnetic flux density and reflecting such variations in Planck constant measurements. Although, the proposed method is very effective in dealing with the asymmetry between the Faraday's and Ampere's Laws, the results would be more rigorous if a comparative study with a compensation coil were conducted. The results of this study indicate that the model fitting the data meets the uncertainty requirement expected in the realisation of a kilogram. However, the accuracy of the measurements is restricted by the PJVS used in the calibration of the 3458A Digital Multimeter, and $0.1 \mathrm{ppm}$ of total relative uncertainty is obtained. The current measurement accuracy will be improved by using differential measurements after integrating PJVS into the measurement set-up. Currently, there are ongoing mechanical modifications being made in our setup to the handler of the mass comparator from which the coil is suspended and the sensors of the magnetic gradiometer. The comparative analysis will be performed after these modifications are completed.

\section{ACKNOWLEDGEMENT}

This research is funded by the TÜBITTAK National Metrology Institute of Turkey.

\section{REFERENCES}

[1] M. J. Milton, R. Davis, N. Fletcher, Towards a new SI: a review of progress made since 2011, Metrologia 51 (2014) pp. R21-30. Online [Accessed 28 September 2020]

DOI: https://doi.org/10.1088/0026-1394/51/3/R21

[2] M. Stock, R. Davis, E. de Mirandés, M. J. T. Milton, The revision of the SI - the result of three decades of progress in metrology, Metrologia 56 (2019) 022001 (14pp). Online [Accessed 28 September 2020] DOI: https://doi.org/10.1088/1681-7575/ab0013

[3] I. A. Robinson, B.P. Kibble, An initial measurement of Planck's constant using the NPL mark II watt balance, Metrologia 44 (2007) pp. 427-40.

DOI: https://doi.org/10.1088/0026-1394/44/6/001

[4] I. A. Robinson, Towards the redefinition of the kilogram: a measurement of the Planck constant using the NPL Mark II watt balance, Metrologia 40 (2012) pp. 113-156.

DOI: http://dx.doi.org/10.1088/0026-1394/49/1/016

[5] A. D. Inglis, C. A. Sanchez, B. M. Wood, The NRC watt balance project, Digest 2010, Conf. on Precision Electromagnetic Measurements, Daejeon, Korea, 13-18 June 2010, pp. 514-515. DOI: https://doi.org/10.1109/CPEM.2010.5543646

[6] C. A. Sanchez, B.M. Wood, R.G. Green, J. O. Liard, D. Inglis, A determination of Planck's constant using the NRC watt balance, Metrologia 51 (2014) pp. S5-S14.

DOI: https://doi.org/10.1088/0026-1394/51/2/S5

[7] R. Steiner, D. Newell, E. Williams, Details of the 1998 Watt balance experiment determining the Planck constant, J. Res. Nat. Inst. Stand. Technol. 110 (2005) pp. 1-26.

DOI: https://doi.org/10.6028/jres.110.003

[8] S. Schlamminger, R. L. Steiner, D. Haddad, D. B. Newell, F. Seifert, L. S. Chao, R. Liu, E. R. Williams, J. R. Pratt, A summary of the Planck constant measurements using a watt balance with a superconducting solenoid at NIST, Metrologia 52 (2015) pp. L5-L8.

DOI: https://doi.org/10.1088/0026-1394/52/2/L5

[9] S. Schlamminger, D. Haddad, F. Seifert, L. S. Chao, D. B. Newell, R. Liu, R. L. Steiner, J. R. Pratt, Determination of the Planck constant using a watt balance with a superconducting magnet system at the National Institute of Standards and Technology, Metrologia 51 (2014) pp. S15-24.

DOI: https://doi.org/10.1088/0026-1394/51/2/S15

[10] D. Haddad, F. Seifert, L. S. Chao, S. Li, D. B. Newell, J. R. Pratt, C. Williams, S. Schlamminger, A precise instrument to determine the Planck constant, and the future kilogram, Rev. Sci. Instrum. 87 (2016) pp. 061301.

DOI: https://doi.org/10.1063/1.4953825

[11] H. Baumann, A. Eichenberger, F. Cosandier, B. Jeckelmann, R. Clavel, D. Reber, D. Tommasini, Design of the new METAS watt balance experiment Mark II, Metrologia 50 (2014) pp. 235-242. DOI: https://doi.org/10.1088/0026-1394/50/3/235

[12] G. Geneves, P. Gournay, A. Gosset, M. Lecollinet, F. Villar, P. Pinot, P. Juncar, A. Clairon, A. Landragin, D. Holleville, F. Pereira Dos Santos, J. David, M. Besbes, F. Alves, L. Chassagne, Suat Topçu, The BNM watt balance project, IEEE Trans. Instrum. Meas. 54 (2005) pp. 850-853.

DOI: https://doi.org/10.1109/TIM.2005.843576

[13] A. Eichenberger, G. Geneves, P. Gournay, Determination of the Planck constant by means of a watt balance, Eur. Phys. J. Spec. Top. 172 (2009) pp. 363-83.

DOI: https://doi.org/10.1140/epjst/e2009-01061-3

[14] C. M. Sutton, An oscillatory dynamic mode for a watt balance, Metrologia 46 (2009) pp. 467-472.

DOI: https://doi.org/10.1088/0026-1394/46/5/010 
[15] C. M. Sutton, M. T. Clarkson, A magnet system for the MSL watt balance, Metrologia 51 (2014) pp. S101-106. DOI: https://doi.org/10.1088/0026-1394/51/2/S101

[16] D. Kim, B.-C. Woo, K.-C. Lee, K.-B. Choi, J.-A. Kim, J. W. Kim, J. Kim, Design of the KRISS watt balance, Metrologia 51 (2014) pp. S96-100.

DOI: https://doi.org/10.1088/0026-1394/51/2/S96

[17] M. Thomas, P. Espel, D. Ziane, P. Pinot, P. Juncar, F. Pereira Dos Santos, S. Merlet, F. Piquemal, G. Genevès, First determination of the Planck constant using the LNE watt balance, Metrologia 52 (2014) pp. 433-443. DOI: http://dx.doi.org/10.1088/0026-1394/52/2/433

[18] M. Thomas, D. Ziane, P. Pinot, R. Karcher, A. Imanaliev, F. Pereira Dos Santos, S. Merlet, F. Piquemal, P. Espel, A determination of the Planck constant using the LNE Kibble balance in air, Metrologia 54 (2017) pp. 468-480. DOI: https://doi.org/10.1088/1681-7575/aa7882

[19] H. Fang, A. Kiss, E. de Mirandés, J. Lan, L. Robertsson, S. Solve, A. Picard, M. Stock, Status of the BIPM watt balance, IEEE Trans. Instrum. Meas. 62 (2013) pp. 1491-1498. DOI: https://doi.org/10.1109/TIM.2012.2225930

[20] S. Li, F. Bielsa, M. Stock, A. Kiss, H. Fang, Coil-current effect in Kibble balances: analysis, measurement, and optimization, Metrologia 55 (2018) pp. 75-83. DOI: https://doi.org/10.1088/1681-7575/aa9a8e

[21] Z. Zhonghua, H. Qing, L. Zhengkun, H. Bing, L. Yunfeng, L. Jiang, L. Chen, L. Shisong, X. Jinxin, W. Nong, W. Gang, G. Hongzhi, The joule balance in NIM of China, Metrologia 51 (2014) pp. S25-31. DOI: https://doi.org/10.1088/0026-1394/51/2/S25

[22] L. Zhengkun, Z. Zhang, Y. Lu, P. Hu, Y. Liu, J. Xu, Y. Bai, T. Zeng, G. Wang, Q. You, D. Wang, S. Li, Q. He, J. Tan, The first determination of the Planck constant with the joule balance NIM-2, Metrologia 54 (2017) pp. 763-774. DOI: https://doi.org/10.1088/1681-7575/aa7a65

[23] I. A. Robinson, S. Schlamminger, The watt or Kibble balance: a technique for implementing the New SI definition of the unit of mass, Metrologia 53 (2016) pp. A46 - A74. Online [accessed 28 September 2020] DOI: https://doi.org/10.1088/0026-1394/53/5/A46

[24] B. Andreas, Y. Azuma, G. Bartl, P. Becker, H. Bettin, M. Borys, I. Busch, M. Gray, P. Fuchs, K. Fujii, H. Fujimoto, E. Kessler, M. Krumrey, U. Kuetgens, N. Kuramoto, G. Mana, P. Manson, E. Massa, S. Mizushima, A. Nicolaus, A. Picard, A. Pramann, O. Rienitz, D. Schiel, S. Valkiers, A. Waseda, Determination of the Avogadro constant by counting the atoms in a ${ }^{28} \mathrm{Si}$ crystal, Phys. Rev. Lett. 106 (2011) pp. 030801

DOI: https://doi.org/10.1103/PhysRevLett.106.030801

[25] B. Andreas, Y. Azuma, G. Bartl, P. Becker, H. Bettin, M. Borys, I. Busch, P. Fuchs, K. Fujii, H. Fujimoto, E. Kessler, M. Krumrey, U. Kuetgens, N. Kuramoto, G. Mana, E. Massa, S. Mizushima, A. Nicolaus, A. Picard, A. Pramann, O. Rienitz, D. Schiel, S. Valkiers, A. Waseda, S. Zakel, Counting the atoms in a ${ }^{28} \mathrm{Si}$ crystal for a new kilogram definition, Metrologia 48 (2011) pp. S1-13. DOI: https://doi.org/10.1088/0026-1394/48/2/S01

[26] Y. Azuma, P. Barat, G. Bartl, H. Bettin, M. Borys, I. Busch, L. Cibik, G. D'Agostino, K. Fujii, H. Fujimoto, A. Hioki, M. Krumrey, U. Kuetgens, N. Kuramoto, G. Mana, E. Massa, R. Meeß, S. Mizushima, T. Narukawa, A. Nicolaus, A. Pramann, S. A. Rabb, O. Rienitz, C. Sasso, M. Stock, R. D. Vocke Jr, A Waseda, S. Wundrack, S. Zakel, Improved measurement results for the Avogadro constant using a ${ }^{28 S i-e n r i c h e d ~ c r y s t a l, ~}$ Metrologia 52 (2015) pp. 360-375. Online [Accessed 28 September 2020]

DOI: https://doi.org/10.1088/0026-1394/52/2/360
[27] G. Mana, E. Massa, C. P. Sasso, The correlation of the $N_{A}$ measurements by counting ${ }^{28} \mathrm{Si}$ atoms, J. Phys. Chem. Ref. Data 44 (2015) pp. 031209.

DOI: https://doi.org/10.1063/1.4921240

[28] K. Fujii, H. Bettin, P. Becker, E. Massa, O. Rienitz, A. Pramann, A. Nicolaus, N. Kuramoto, I. Busch, M. Borys, Realization of the kilogram by the XRCD method, Metrologia 53 (2016) pp. A19-45. Online [Accessed 28 September 2020]

DOI: https://doi.org/10.1088/0026-1394/53/5/A19

[29] N. Kuramoto, S. Mizushima, L. Zhang, K. Fujita, Y. Azuma, A. Kurokawa, S. Okubo, H. Inaba, K. Fujii, Determination of the Avogadro constant by the XRCD method using a ${ }^{28}$ Si-enriched sphere, Metrologia 54 (2017) pp. 716-729. Online [Accessed 28 September 2020]

DOI: https://doi.org/10.1088/1681-7575/aa77d1

[30] B. P. Kibble, A Measurement of the gyromagnetic ratio of the proton by the strong field method, in: Atomic Masses and Fundamental Constants, vol. 5. J. H. Sanders, A. H. Wapstra (editors), New York: Plenum, 1976, pp 545-551.

[31] B. P. Kibble, I. A. Robinson, Feasibility study for a moving coil apparatus to relate the electrical and mechanical SI units, Technical Report DES 40 (1977) NPL.

[32] K. von Klitzing, G. Dorda, M. Pepper, New method for highaccuracy determination of the fine-structure constant based on quantized Hall resistance, Phys. Rev. Lett. 45 (1980) pp. 494-497. DOI: https://doi.org/10.1103/PhysRevLett.45.494

[33] B. D. Josephson, Possible new effects in superconductive tunnelling, Phys. Lett. 1 (1962) pp. 251-253. DOI: https://doi.org/10.1016/0031-9163(62)91369-0

[34] H. Ahmedov, An Oscillating magnet watt balance, 2016 Conference on Precision Electromagnetic Measurements (CPEM 2016), Ottawa, Canada, 10-15 July 2016. DOI: https://doi.org/10.1109/CPEM.2016.7540780

[35] H. Ahmedov, N. Babayiğit Așkın, B. Korutlu, R. Orhan, Preliminary Planck constant measurements via UME oscillating magnet Kibble balance, Metrologia 55 (2018) pp. 326-333. DOI: https://doi.org/10.1088/1681-7575/aab23d

[36] H. Ahmedov, B. Korutlu, R. Orhan, Optimization Procedure for Faraday's Voltage in UME Kibble Balance, 2018 Conference on Precision Electromagnetic Measurements (CPEM 2018), Paris, France, 8-13 July 2018. DOI: https://doi.org/10.1109/CPEM.2018.8500794

[37] H. Ahmedov, B. Korutlu, R. Orhan, Optimization Procedure for Faraday's Voltage in UME Kibble Balance, IEEE Transactions on Instrumentation and Measurement 68 no. 6 (2019) pp. 2172-2175. DOI: https://doi.org/10.1109/TIM.2019.2899185

[38] H. Fang, F. Bielsa, S. Li, A. Kiss, M. Stock, The BIPM Kibble balance for the realization of the redefined kilogram, 2018 Conference on Precision Electromagnetic Measurements (CPEM 2018), Paris, France, 8-13 July 2018. DOI: https://doi.org/10.1109/CPEM.2018.8500899

[39] S. Schlamminger, Design of the Permanent-Magnet System for NIST-4, IEEE Trans. Instrum. Meas. 62 (2012) pp. 1524-1530. DOI: https://doi.org/10.1109/TIM.2012.2230771

[40] Z. Zhang, Z. Li, B. Han, Y. Lu, S. Li, J. Xu, G. Wang, Coils and the Electromagnet Used in the Joule Balance at the NIM, IEEE Trans. Instrum. Meas. 64 (2015) pp. 1539-1545. DOI: https://doi.org/10.1109/TIM.2015.2406055

[41] L. Zhengkum, Y. Bai, J. Xu, Q. You, D. Wang, Z. Zhang, Y. Lu, P. Hu, Senior Member, IEEE, Y. Liu, Q. He, J. Tan, The Improvements of the NIM-2 Joule Balance, IEEE Transactions on Instrumentation and Measurement 68 no. 6 (2019) pp. 22082214.

DOI: https://doi.org/10.1109/TIM.2019.2893717 